\title{
Seizures using a nasal decongestant
}

\section{Crises comiciais usando um descongestionante nasal}

To the editor:

Posterior reversible encephalopathy syndrome (PRES) is a well-known clinicoradiological syndrome characterized by severe headache with or without neurological deficits or seizures and edema of subcortical white matter in diverse brain areas $^{1,2}$. The predominant involvement of posterior cerebral lobes may be explained by an augmented vulnerability of this zones in part due to decreased sympathetic innervation and subsequent hyperperfusion and vasogenic edema ${ }^{3}$.

We report a 47 year old caucasian woman with no previous medical problems, admitted to the emergency department for acute onset of generalized tonic-clonic seizures. The patient also presented complaints of holocranial headache, nausea and vomiting that persisted with fluctuating severity for the two previous weeks. One month prior to admission she was diagnosed with allergic rhinitis and treated with a nasal decongestant containing pseudoephedrine applied twice a day since then. On admission she had elevated blood pressure over 180/100 $\mathrm{mmHg}$, normal heart rate and was afebrile. On neurologic examination she was conscient, oriented, with right homonymous hemianopsia, right hemiparesis (MRC 4/5) and ipsilateral babinsky sign being the remainder neurologic examination normal. The full blood count, sedimentation rate, C-reactive protein, immunologic and thrombotic studies, viral serologies, CSF analysis with neurotropic virus were normal or negative. Head CT revealed bilateral parieto-occipital lobe hypodensities and brain MRI confirmed abnormal T2 and FLAIR signal and acute water restriction in DWI in the same location.

Due to the suggestive clinical and imaging picture, PRES diagnosis was established. Nasal pseudoephedrine was suspended and the patient was treated with anti-hypertensive and antiepileptic drugs with subsequent lowering of blood pressure and no recurrence of seizures. The study of hypertension failed to find any etiologic cause other than the use of pseudoephedrine. She was discharged two weeks later with normal blood pressure, normal neurologic exam and follow-up brain MRI with complete resolution of white matter abnormalities.

Hypertension and some medications such as chemotherapeutic agents, imunossupressive, cytotoxic treatments for malignant neoplasms or immunologically based conditions have been linked to PRES ${ }^{4}$. However the use of pseudoephedrine has been infrequently reported as a precipitating factor for this syndrome ${ }^{5}$.

Pathophysiology is incompletely understood, but sympathicomimetic agents such as pseudoephedrine present in some nasal decongestants may play a role in PRES by promoting elevation of blood pressure, a side effect of sympathicomimetic drugs ${ }^{5}$. Elevated blood pressure and failure of cerebral autoregulation by several mechanisms can lead to cerebral edema and ultimately precipitate $\mathrm{PRES}^{6}$.
The authors report a case of PRES most likely secondary to the use of pseudoephedrine. Thorough investigation excluded other etiologies such as vasculitis or stroke and left pseudoephedrine as the only trigger event identified.

\section{References}

1. Call GK, Fleming MC, Sealfon S, et al. Reversible cerebral segmental vasoconstriction. Stroke. 1988; 19: 1159-70.

2. Chen S., Fuh J., Wang S. Reversible cerebral Vasoconstriction syndrome: an under-recognized clinical emergency. Ther Adv Neurol Disord. 2010; 3(3) 161-71.

3. Fugate J., Claassen D., Cloft H., Kallmes D, Kozak O., Rabinstein A. Posterior Reversible Encephalopathy Syndrome: Associated Clinical and Radiologic Findings. Mayo Clin Proc. May 2010; 85(5):427-32

4. W.S. Bartynski. Posterior Reversible Encephalopathy Syndrome, Part 2: Controversies Surrounding Pathophysiology of Vasogenic Edema. AJNR Am J Neuroradiol 2008: 29:1043- 49.

5. M. Ebbo, L. Benarous, G. Thomas, N. Jourde, S. Genot, E. Bernit, V. Veit, J.-R. Harlé, N. Schleinitz. Syndrome d'encéphalopathie postérieure reversible induit par un décongestionnant nasal contenant de la pseudo-éphédrine. La revue de médecine interne 2009: 10.436

6. Hedna V., Stead L., Bidari S., Patel A., Gottipati A., Favilla C., Salardini A., Khaku A., Mora D., Pandey A., Patel H., Waters M. Posterior reversible encephalopathy syndrome (PRES) and CT perfusion changes. International Journal of Emergency Medicine 2012, 5:12.

Note: this letter was partially delivered as a poster at the $7^{\circ}$ Portuguese Congress of Stroke. Oporto, Portugal, February 2013

Figure 1. On second week of symptoms. 1- Head CT shows bilateral occipital hipodensities; 1'- Brain MRI imaging reveals bilateral parieto occipital white matter areas compatible with edema, hyperintense in FLAIR $\left(1^{\star}\right)$ and T2 $\left(1^{\star *}\right)$.

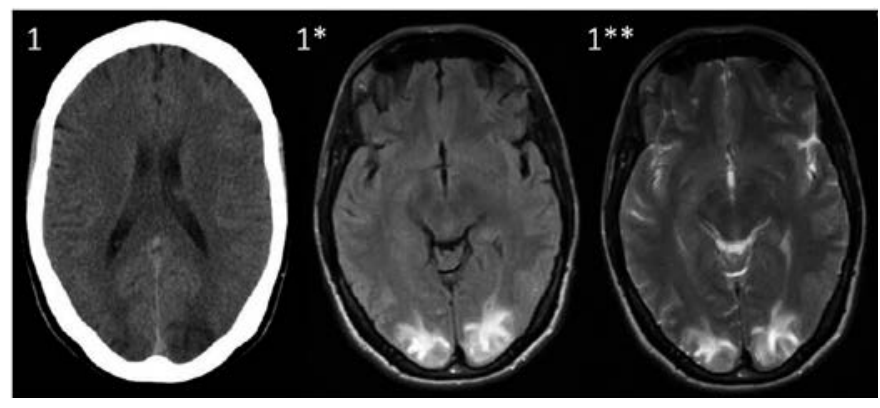

Figure 2. On fourth week of illness. 2' - Brain MRI with complete resolution of white matter lesions on FLAIR $\left(2^{\star}\right)$ and T2 $\left(2^{\star \star}\right)$.

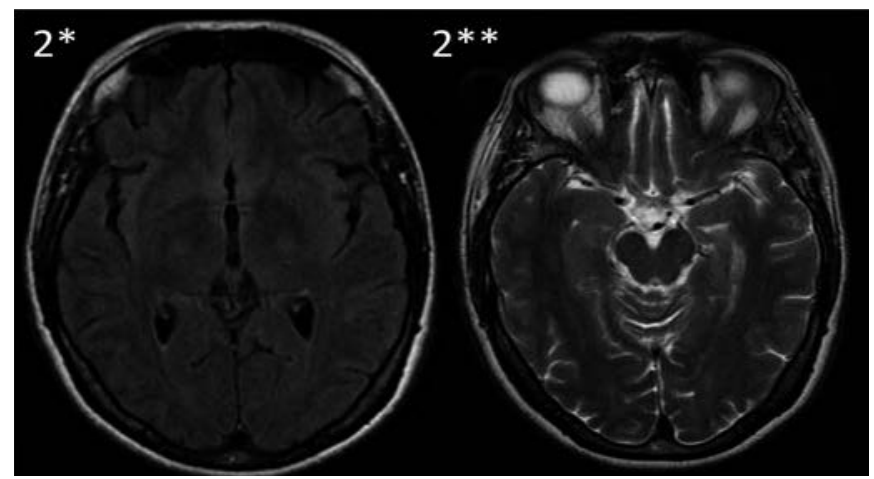

Sílvia César, Sandra Perdigao

Neurology Department. Unidade Local de Saúde do Alto Minho. Viana do Castelo. Portugal 
lavado con soluciones cloradas ${ }^{4,5}$. Con esta simple medida la mortalidad materna en la Clínica 1 disminuyó abruptamente y se mantuvo baja durante los años siguientes.

A pesar de la evidencia experimental la teoría fue rechazada por los líderes de opinión más influyentes del momento, se la acusaba a Semmelweis de que carecía de soporte racional y de que el lavado de manos era una teoría demasiado simplista. El tiempo y la epidemiología acabarían dando la razón al médico húngaro, con el cual la medicina ha adquirido una deuda impagable.

Han pasado 150 años desde el fallecimiento de SemmeIweiss pero el mal uso de la higiene de manos persiste hoy en día, teniendo una adherencia baja por parte del personal sanitario. En esta línea, un grupo de investigadores suecos ${ }^{6}$ realizó un estudio para analizar la adherencia a la higiene de manos durante la práctica anestésica. Observaron que la adherencia a las guías era tan sólo de un 5.3\%, con variaciones según el tipo de intervención quirúrgica (mayor en las traumatológicas $(7.1 \%)$ y menor en las urológicas (3.2\%)). Además, los autores observaron que la higiene de manos era más frecuente después de la realización de un procedimiento que antes del mismo.

La importancia de la higiene de manos se ha puesto de relieve en varias publicaciones como medida esencial para evitar infecciones/colonizaciones por gérmenes multirresistentes ${ }^{7}$, además se ha constatado que su realización tiene un impacto mayor que un programa de cribado generalizado o programas de vigilancia complejos en la prevención de la infección nosocomial en los centros sanitarios de cuidados prolongados ${ }^{8}$.

\section{Blbliografía}

1. Best M, Neuhauser D. Ignaz Semmelweis and the birth of infection control. Qual Saf Health Care 2004; 13: 233-4.

2. Lee R, Chien A. Semmelweis. Med Hist 2005; 48: 616-8

3. Nuland SB. The enigma of Semmelweis-an interpretation. J Hist Med Allied Sci 1979;34.:255-272

4. Gargantilla P. Manual de Historia de la Medicina. $3^{\circ}$ edición. Grupo Editorial33. Málaga, 2013.

5. Noakes TD, Borresen J, Hew-Butler T, Lambert MI, Jordaan E. Semmelweis and the aetiology of puerperal sepsis 160 years on: and historical review. Epidemiol Infect 2008; 136: 1-9

6. Megeus V, Nilsson K, Karlsson J, Eriksson B, Andersson AE. Hand hygiene and aseptic techniques during routine anesthesic care-observations in the operating room. Antimicrobial Resistance and Infection Control 2015; 4:5 D0l:10.1186/s13756015-0042-y

7. Rodíguez E, Díaz B. Infecciones/colonizaciones por Gérmenes Multirresistentes. Galicia Clin 2014;75(1):17-21

8. Serrano M, Barcenilla F, Limón E. Infección nosocomial en centros sanitarios de cuidados prolongados. Enferm Infecc Microbiol Clin 2014;32(3):191-8.

\section{Pedro Gargantilla Madera ${ }^{1,2}$, Noelia Arroyo Pardo ${ }^{1}$, Emilio Pintor Holguin ${ }^{2}$}

${ }^{1}$ Hospital de El Escorial de Madrid, ${ }^{2}$ Universidad Europea de Madrid

\section{Normas de publicación Galicia Clínica}

Galicia Clínica es la revista oficial de la Sociedad Gallega de Medicina Interna (SOGAMI). Se publican 4 números al año, simultáneamente en papel y en www.galiciaclinica. info, incluyéndose en el segundo de ellos las comunicaciones enviadas a la correspondiente Reunión Ordinaria de la SOGAMI.

\begin{abstract}
GALICIA CLÍNICA evaluará para su publicación trabajos médicos relacionados preferentemente con la Medicina Interna y sus subespecialidades, y/o con problemas médicos prevalentes en la Comunidad Autónoma de Galicia.

Se admitirán para evaluación trabajos en castellano, gallego, inglés y portugués.

Para el envío de originales se ha habilitado un formulario en la pagina web www.galiciaclinica.info. El sistema confirmarála entrega y permitirá consultar el estado del manuscrito. No se aceptarán originales enviados por otros métodos.

El comité editorial, eventualmente con la ayuda de revisores externos, evaluará los trabajos enviados decidiendo si procede su publicación, si es necesario realizar correcciones o si se desestima la publicación. Una vez aceptado, se enviarán a autor las pruebas de imprenta para la corrección de posibles erratas.

Los trabajos reunirán los requisitos de uniformidad habituales en revistas biomédicas. Dichos requisitos se pueden consultar en "Uniform Requirements for Manuscripts Submitted to Biomedical Journals: Writing and Editing for Biomedica Publication, Updated April 2010", disponible en http://mww.icmje.org. Se recomienda encarecidamente leer en especia la sección "Preparing a Manuscript for Submission to a Biomedical Journal" (http://wwww.icmje.org/manuscript_1 prepare. html) y seguir fielmente sus indicaciones a la hora de redactar el trabajo a enviar.

Se recomienda el empleo de los programas más habituales de edición de texto (Ej., Word) tanto para el texto como para las tablas.

Dado que la mayoría de las páginas se imprimen en blanco y negro, se aconseja evitar en tablas y figuras en la medida de lo posible el uso de colores o tramas que no tengan el adecuado contraste para su identificación.

Las figuras o imágenes se enviarán en archivo aparte, como archivo de imagen (jpeg o similar) o como PDF con una resolución de 300 ppp. a tamaño de impresión definitivo.
\end{abstract}

La revista presenta las siguientes secciones:

Editoriales

Habitualmente encargados por la dirección de la revista. Su extensión máxima será de 8 páginas de 30 líneas y se admitira una figura 0 una tabla y quince citas bibliográficas.

El número máximo de firmantes será de dos.

Originales

Trabajos de investigación sobre cualquier aspecto médico. La estructura general de los trabajos será la tradicional: Título: en el idioma original y en inglés, Resumen y Abstract en inglés, Palabras clave y Key words (que deben corresponder a los medical subjets headings -MESH- del Index Medicus), Introducción, Material y métodos, Resultados, Discusión, Bibliografía La extensión máxima recomendada del texto es de 20 páginas de 30 líneas, a las que se podrán añadir 5 figuras y 5 tablas, y un máximo de 30 citas bibliográficas.

El número máximo de firmantes será de ocho.

Originales breves

Trabajios de investigación que por sus características no precisan un mayor espacio. Estructura similar a la de los originales. Su extensión máxima será de 10 páginas de 30 líneas, 3 figuras, 3 tablas y 20 citas bibliográficas. El número máximo de firmantes será de seis.
Revisiones

Habitualmente encargadas por la dirección de la revista. La extensión máxima recomendada del texto es de 30 páginas de 30 lineas, a las que se podrán añadir 6 figuras y 6 tablas, y un máximo de 50 citas bibliográficas. El número máximo de firmantes será de tres.

Preguntas clínicas

En esta sección se tratará de responder de forma concreta y sucinta a preguntas clínicas concretas que, bien han motivado una controversia, o cuestionan actitudes arraigadas en la práctica diaria. La extensión máxima será de 6 páginas de 30 líneas, dos figuras y dos tablas y 15 citas bibliográficas.

I número máximo de firmantes será de dos.

Notas clínicas

Descripción de casos clínicos de excepcional interés. Constarán de una breve introducción, caso clinico, y discusión correspondiente. Su extensión máxima será de 6 páginas, 2 figuras y dos tablas y 15 citas bibliográficas.

El número máximo de firmantes será de cuatro.

Cartas al director

Comentarios, opiniones u observaciones sobre los diversos trabajos publicados con anterioridad en la revista. La extensión máxima será de 4 páginas de 30 líneas y se admitirá una figura 0 una tabla y diez citas bibliográficas.

El número máximo de firmantes será de dos.

Imágenes médicas

Imágenes curiosas, insólitas o demostrativas. Se acompañarán con un texto breve, como máximo 1 página de 30 líneas, en el que se explique el caso clínico, con una breve discusión acerca de la importancia de la imagen.

El número máximo de firmantes será de dos.

Resúmenes de Tesis doctorales

Elaborados por el autor, describirán el trabajo realizado; su extensión máxima será de 2 páginas de 30 líneas. Debe incluise un apéndice con los datos correspondientes a Universidad, departamento, director de la tesis y fecha de presentación. Otros

La dirección de la revista considerará para su publicación cualquier artículo relacionado con la medicina en cualquier aspecto, aunque no se incluya exactamente dentro de los supuestos anteriores. En este caso se recomienda antes de su envio contactar con la dirección para acordar las características del mismo.

En el caso de que los trabajos enviados incluyan imágenes, figuras, tablas o textos sometidos a copyright, será responsabilidad de los autores la obtención de los permisos necesarios para su publicación.

Todas las opiniones o afirmaciones expresadas en los artículos corresponden a los autores de los mismos. Tanto el comité editorial como la SOGAMI declinan cualquier responsabilidad a este respecto.

Los trabajos publicados serán propiedad de GALCIA CLINICA, cediendo los autores todos los derechos a la misma. 